\title{
Magnetohydrodynamic parametric instabilities of parallel propagating incoherent Alfvén waves
}

\author{
Yasuhiro Nariyuki and Tohru Hada
}

E.S.S.T., Kyushu University, Japan

(Received December 4, 2006; Revised January 10, 2007; Accepted January 16, 2007; Online published March 28, 2007)

\begin{abstract}
Numerical experiments for parametric instabilities of incoherent Alfvén waves in the context of one dimensional Hall-MHD equation sets are studied. The reason why the decay instability of incoherent waves can be explained in terms of that of the coherent wave is understood through analysis on nonlinearly driven finite amplitude density fluctuations. Numerical results suggest the importance of modulational instabilities of left-hand polarized Alfvén waves.
\end{abstract}

Key words: Alfvén waves, solar wind.

\section{Introduction}

Large amplitude Alfvén waves are ubiquitous in the solar wind (Bruno and Carbone, 2005; Goldstein et al., 2005), and are believed to play important roles in the acceleration and heating of the solar wind plasma (see Suzuki and Inutsuka, 2006). The solar wind Alfvén waves are typically robust for linear ion-cyclotron damping (due to small wave frequencies) and also for linear Landau damping (due to the small propagation angle relative to the background magnetic field). On the other hand, through parametric instabilities, they can transfer their energy into longitudinal waves, which subsequently heat the background plasma through the ion Landau damping.

Parametric instabilities of a parallel propagating coherent (monochromatic) Alfvén wave have been investigated both theorically (Champeaux et al., 1999; Nariyuki and Hada, 2006a, b, and references therein) and numerically (Terasawa et al., 1986; Hoshino and Goldstein, 1989; Agim et al., 1995; Vasquez, 1995; Del Zanna et al., 2001; Turkmani and Torkelsson, 2003; Laveder et al., 2003; Bugnon et al., 2004). The decay instability of incoherent (broad band) Alfvén waves was first considered by Umeki and Terasawa (1992), who numerically studied evolution of the instability starting from a power-law-type spectrum, as actually observed (Tu and Marsch, 1995; Narita et al., 2006). They found that the decay of the waves can be consistently explained by one of the coherent waves when the plasma $\beta$ (=the squared ratio between the sound velocity to the Alfvén velocity) is small. Malara and Velli (1996) subsequently developed a formulation for the decay instability of incoherent Alfvén waves, and Malara et al. (2000) discussed the nonlinear evolution and saturation of the instability without the Hall effect. Agim et al. (1995) discussed the decay instability of the right-hand polarized incoherent

Copy right(c) The Society of Geomagnetism and Earth, Planetary and Space Sciences (SGEPSS); The Seismological Society of Japan; The Volcanological Society of Japan; The Geodetic Society of Japan; The Japanese Society for Planetary Sciences; TERRAPUB. dispersive Alfvén waves and compared the numerical results with observations.

The motivation of the present study is to clarify the reason why the decay instability of incoherent waves can be understood in a manner consistent with that of the coherent wave. We perform simple numerical experiments in the context of one-dimensional Hall-MHD equation sets and explain the mechanism of parametric instabilities of incoherent left-hand polarized waves as a consequence of instabilities nonlinearly driven by finite amplitude density fluctuations. We show the numerical results in Section 2 and summarize the results and discuss some future issues in Section 3.

\section{Numerical Results \\ 2.1 Basic equations}

We consider a set of one-dimensional Hall-MHD equations,

$$
\begin{aligned}
& \frac{\partial \rho}{\partial t}=-\frac{\partial}{\partial x}(\rho u) \\
& \frac{\partial u}{\partial t}=-u \frac{\partial u}{\partial x}-\frac{1}{\rho} \frac{\partial}{\partial x}\left(p+\frac{|b|^{2}}{2}\right) \\
& \frac{\partial v}{\partial t}=-u \frac{\partial v}{\partial x}+\frac{1}{\rho} \frac{\partial b}{\partial x} \\
& \frac{\partial b}{\partial t}=-\frac{\partial}{\partial x}\left(u b-v+\frac{i}{\rho} \frac{\partial b}{\partial x}\right)
\end{aligned}
$$

where $b=b_{y}+i b_{z}$ and $v=v_{y}+i v_{z}$ are the complex transverse magnetic field and velocity, respectively, $u=v_{x}$ is the longitudinal velocity, and other notations are standard. All of the normalizations have been made using the background constant magnetic field, density, Alfvén velocity, and ion gyro-frequency, all defined at a certain reference point. For simplicity, we assume the equation of state to be isothermal, $p=\beta \rho$.

We numerically solved Eqs. (1)-(4) using the rationalized Runge-Kutta scheme for time integration and the spectral method for evaluating spatial derivatives under periodic 
boundary conditions. In order to handle the formation of strong shocks, we have included in the set of equations small dissipation terms (proportional to the second order spatial derivatives, with coefficients $\sim 10^{-4}$ to $10^{-3}$ ). Unless otherwise noted, the number of grid points is 2048 with cell spacing 0.25 .

The initial Alfvén waves are given as

$$
F_{p}=\sum_{k_{0}=k_{1}}^{k_{2}} F_{k_{0}}^{h} \exp \left(i\left(\omega_{0} t-k_{0} x+\phi_{k_{0}}\right)\right)
$$

where $F=b, v, \omega_{0}^{2}=k_{0}^{2}\left(1+\omega_{0}\right), v_{k_{0}}^{h}=-k_{0} b_{k_{0}}^{h} / \omega_{0}$, and $h=r, l$ stands for the right-hand (RH) and left-hand (LH) polarizations, respectively. We adopt the notation that the positive (negative) $\omega_{0}$ corresponds to the $\mathrm{RH}(\mathrm{LH})$ polarized waves. The initial waves are given within the interval of $k_{1}$ and $k_{2}$ (the case $k_{1}=k_{2}$ corresponds to the coherent wave). The phase $\phi_{k_{0}}$ is given as random.

\subsection{Coherent Alfvén wave and density mode}

Before running the simulation with incoherent Alfvén waves, we first show the results of a simple numerical experiment on "nonlinear" parametric instabilities, using a coherent finite amplitude Alfvén wave $\left(k_{0}=k_{1}=k_{2}\right)$, small amplitude white noise (the r.m.s. amplitude $=10^{-5}$ for all the variables), and a finite amplitude monochromatic sound wave, $\rho_{0} \exp \left(i\left(\Omega_{0} t-K_{0} x\right)\right.$ ), where $\Omega_{0} / K_{0}= \pm \sqrt{\beta}$ (we choose here +$)$.

Figure 1(a) and (b) shows time evolution of the magnetic field power spectrum. The parameters used are, for both of the figures, $\beta=0.4, b_{k_{0}}^{r}=0.3, b_{k_{0}}^{l}=0, k_{0}=0.49, \omega_{0}=$ $0.63, \rho_{0}=0.1$, and $K_{0}=0.60$ for Fig. 1 (a) and $K_{0}=0.65$ for Fig. 1(b). Superposed within the figures are the linear growth rate (assuming that finite amplitude sound wave is absent). The coupling between the initial Alfvén wave at $k=k_{0}$ and the initial sound wave at $k=K_{0}$ produces a 'seed' of transverse daughter waves at $k_{i}=k_{ \pm}=k_{0} \pm K_{0}$, which we will call Initially Coupled Mode (ICM) hereafter. If the ICM happens to be within the decay unstable regime, it is preferentially amplified, as shown in Fig. 1(b). In contrast, if $k_{i}$ is outside the decay unstable regime, the wave at $k_{i}$ gradually ceases away by the dissipation, while the daughter wave corresponding to the maximum growth of the decay instability grows gradually. This is seen in Fig. 1(a), where $k_{i}$ approximates -0.1 and $k$ approximates -0.2 .

The wave at the maximum growth does not become dominant in Fig. 1(b) since much of the parent wave energy is used in the growth of the ICM. The results here indicate that the finite amplitude density fluctuations can nonlinearly control the final (much longer than the linear growth time scale) state of the decay instability.

A similar argument holds for the modulational instability as well. In general, the fate of the instability is determined by relative amplitude of the initial trigger ( $\rho_{0}$ of the ICM in the case discussed above) compared with that of the noise.

\subsection{Incoherent Alfvén waves}

When incoherent Alfvén waves are initially given as a broadband power-law type spectrum (see Umeki and Terasawa (1992), and also the numerical experiments below), density fluctuations are instantaneously produced due to en-
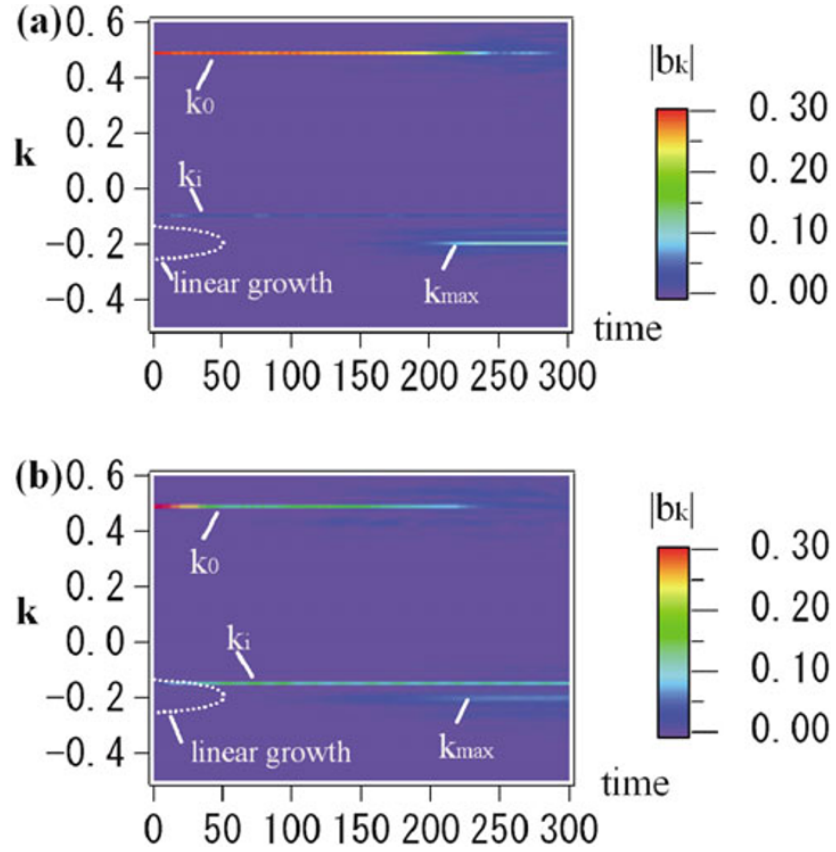

Fig. 1. Time evolution of magnetic field power spectrum (Alfvén waves). Linear growth rate $\times 10^{3}$ of the decay instability is superposed as white dotted lines. Parameters used are: $\beta=0.4, b_{k 0}^{r}=0.3, b_{k 0}^{l}=0$, $k_{0}=0.49, \omega_{0}=0.63, \rho_{0}=0.1$, and (a) $K_{0}=0.59$, so that $k_{i}=k_{0}-K_{0}=-0.10$, and (b) $K_{0}=0.64, k_{i}=-0.15$. In (a) the daughter Alfvén wave around the maximum linear growth is amplified eventually, while in (b) instead, the initially coupled mode (ICM), triggered due to the presence of the parent Alfvén and the sound waves, is preferentially amplified.

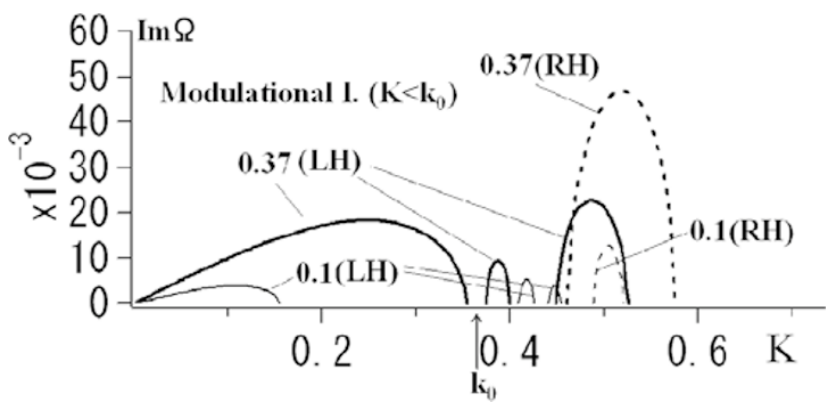

Fig. 2. The linear growth rates of parametric instabilities plotted versus $K$, the wave number of the daughter density fluctuations. Parameters used are: $\beta=0.35$ for all the cases, $b_{k_{0}}^{h}=0.1$ (thin lines) and 0.37 (thick lines), and $k_{0}=0.37$. Dashed and solid lines represent the cases with the R.H. $(h=r)$ and L.H. $(h=l)$ polarized parent wave. The wave numbers of the daughter Alfvén waves are given as $k_{0} \pm K$.

velope modulation of the magnetic field (see Agim et al., 1995). Such density modes are usually stable to the decay instability owing to their small wave numbers (see Vasquez, 1995), and thus they do not play major roles in the nonlinear evolution of the instability. This is the main reason why the decay of incoherent Alfvén waves remains essentially the same as that of the coherent Alfvén wave (Umeki and Terasawa, 1992). Another point of practical importance is that the unstable region of the decay instability is often quite narrow in the wave number space in numerical simulations: as an example, we compare the decay instability $\left(k_{0}<K\right.$, where $K$ is the wave number of longitu- 


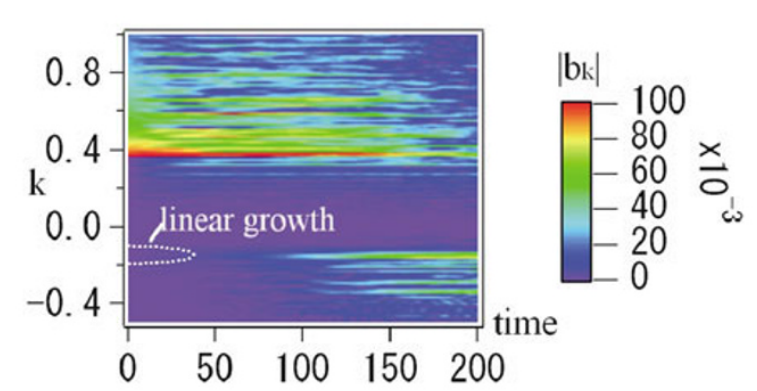

Fig. 3. Numerical simulation of the R.H. polarized incoherent Alfvén waves. Shown is the time evolution of the magnetic field power spectrum. Parameters used are: $\beta=0.35, k_{1}=0.37, k_{2}=1.1$, $\left|b_{k_{0}}^{r}\right|^{2} \propto|k|^{-3}$, and $b_{k_{1}}^{r}=0.1$. The linear growth rate $\times 10^{3}$ of the decay instability for the parent wave at $k_{0}=0.37, b_{k_{0}}^{r}=0.37$ is superposed. Evolution of the incoherent Alfvén waves can be explained using the linear growth of the coherent wave (thin and thick dashed lines in Fig. 2): it is seen that the daughter Alfvén wave with the wave number $k \sim-0.15 \sim k_{1}-K_{\max }$ is dominantly excited.

dinal fluctuations) and modulational instability $\left(k_{0}>K\right)$ growth rates in Fig. 2. When the system size is 256 and $b_{k 0}$ approximates 0.1 (thin lines), values typically used in previous numerical studies, the regime with positive decay growth includes only a few modes, and the nonlinear coupling we argued above may not be realized simply due to the lack of enough spatial resolutions. As shown in Fig. 2, when $\beta<1$ in the fluid system, the RH polarized waves are unstable only to the decay instability, while the LH polarized waves are unstable to both the decay and modulational instabilities (see Champeaux et al., 1999).

Figure 3 shows the time evolution of the magnetic field power spectrum for $\beta=0.35, k_{1}=0.37, k_{2}=1.1$, $\left|b_{k_{0}}^{r(l)}\right|^{2} \propto|k|^{-3}$, and $b_{k_{1}}^{r}=0.1$. The linear growth due to the Alfvén wave at $k_{0}=k_{1}$ (the thick dashed line in Fig. 2) is superposed. As expected, the decay of the RH polarized incoherent Alfvén waves take place in a manner consistent with that of the coherent wave. The daughter Alfvén wave at $k \sim-0.15 \sim k_{1}-K_{\max }$ is the most dominantly excited.

The LH polarized incoherent Alfvén waves evolve quite differently (Fig. 4). Since the density fluctuations produced by the envelope modulation are unstable to the modulational instability (the thick solid line in Fig. 2), they grow using much of the energy originally stored in the initial Alfvén waves, leaving the decay instability to be inactive even though it has the largest linear growth rate.

Finally, we remark that the finite correlation among wave phases is more strongly generated by the modulational instability than by the decay instability. From the comparison of structure functions of a given wave data and that of the phase-shuffled/phase-correlated surrogates, a method to quantitatively evaluate the phase coherence among waves has been proposed and applied to foreshock MHD waves (Hada et al., 2003). We have applied the method to the magnetic field data produced by the decay and the modulational instabilities shown in Figs. 3 and 4. The phase coherence index $\left(C_{\phi}\right)$ being close to 0 indicates that the waves phases are almost randomly distributed, while larger values of $C_{\phi}$ imply the presence of phase coherence. Figure 5 shows time

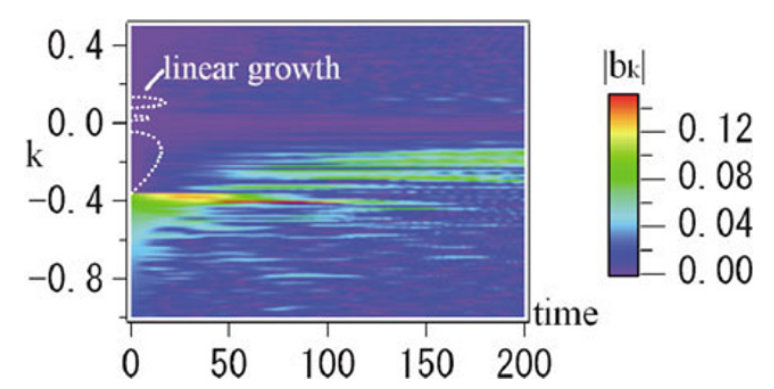

Fig. 4. The same as Fig. 3, but for the L.H. polarized incoherent Alfvén waves, which propagate in the same direction as the initial R.H. waves in Fig. 3. Parameters used are: $\beta=0.35, k_{1}=-0.37, k_{2}=-1.1$, $\left|b_{k_{0}}^{l}\right|^{2} \propto|k|^{-3}$, and $b_{k_{1}}^{l}=0.1$. Evolution of incoherent Alfvén waves cannot be explained using the linear growth of the coherent wave (thick and thin solid lines in Fig. 2). Rather than the decay instability $(k>0)$, the modulational instability $(k<0)$ becomes dominant, as it is nonlinearly triggered by the density fluctuations produced by the magnetic field envelope modulation, even though the linear growth rate for the decay is larger than that of the modulational instability.

evolution of $C_{\phi}$ for the run shown in Fig. 3 (black) and in Fig. 4 (gray). The phase coherence is clearly produced as a consequence of the modulational instability, while that of the decay instability appears to be weak. This difference comes from the long-range correlation in the Fourier space among wave modes through the modulational instability (see figure 13 in Nariyuki and Hada, 2006a). We point out that finite phase correlation among waves are generated in the solar wind (Hada et al., 2003).

In the solar wind, density fluctuations also consist of power-law-type broadband spectrum (Tu and Marsch, 1995). In parametric instabilities of incoherent Alfvén waves, the density modes with the wave number unstable to the instabilities mainly play important role in the dissipation of Alfvén waves. In addition, whatever the finite phase correlations considered in the initial transverse and longitudinal the present results do not change.

\section{Summary and Discussion}

By performing one-dimensional Hall-MHD simulations, we have studied parametric instabilities of parallel propagating incoherent Alfvén waves and shown that the instabilities are strongly influenced by the presence of finite amplitude density fluctuations, as they can be the seed population for the instabilities. In particular, the incoherent Alfvén waves with broadband power-law-type spectrum can drive modulational instability more dominantly than the decay instability, even if the growth rate of the latter is larger than the former, since the density fluctuations produced by the magnetic field envelope modulation are likely to fall within the modulationally unstable regime in the wave number space.

Such 'nonlinear' instabilities (driven by the finite amplitude density fluctuations and finite amplitude Alfvén waves) are especially important when ion kinetic effects are considered. Although the density fluctuations are strongly damped by ion Landau resonance, the ion kinetic effects expand the decay unstable regime in the wave number space (Vasquez, 1995; Araneda, 1999; Nariyuki and Hada, 2006b). Both the RH and LH polarized waves become unstable to the modu- 


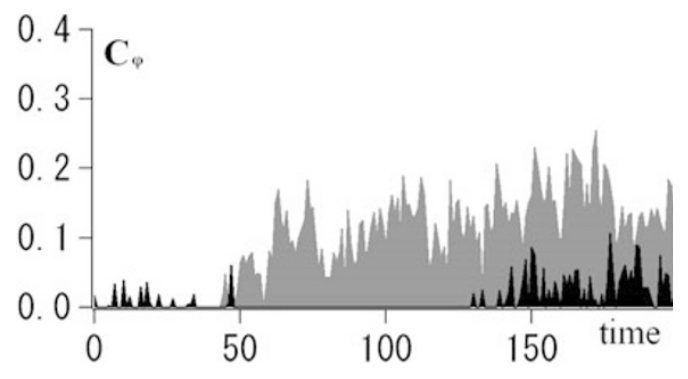

Fig. 5. Comparison of time evolution of the phase coherence index, $C_{\phi}$, evaluated for the run shown in Fig. 3 (black) and Fig. 4 (gray). The modulational instability produces the phase coherence more effectively than the decay instability.

lational instability in a finite beta plasma such as the solar wind, and the nonlinearly driven modulational instability such as those shown Fig. 4 should be taking place in reality. The presence of the phase coherence among large amplitude foreshock MHD waves (Hada et al., 2003) may be ascribed to the modulational type instabilities, rather than the decay.

The maximum linear growth rate of the instability obtained from the dispersion relation is of the order of approximately $0.1\left|\Omega_{0}\right|$, while our simulation suggests that the time scale for the daughter waves to grow to a 'visible' amplitude is somewhat (by a factor of two to three) shorter than the reciprocal of the linear growth rate because of the presence of finite amplitude initial seed. In real scale, $\Omega_{i} / 2 \pi$ is $\sim 0.1$ $\mathrm{Hz}$ in the solar wind near $1 \mathrm{AU}$ and $\sim 10^{3} \mathrm{~Hz}$ in the solar corona, so that the instability time scale can be roughly estimated at $\sim 30 \mathrm{sec}$ and $\sim 10^{-3} \mathrm{sec}$, respectively, suggesting that the parametric processes may be important in the dissipation of Alfvén waves in both of these regions. However, other processes such as the linear wave conversion due to background field/plasma inhomegeneity may be important as well, in particular in the coronal heating by Alfvén waves (see Suzuki and Inutsuka, 2005; Tsiklauri et al., 2005).

Finally, we list the limitations of the present work. We have considered a one-dimensional system, assuming that the initially quasi-parallel propagating waves remain to be so at long (compared with linear growth) time scales. The assumption breaks down when the background is inhomogeneous, or the coupling to oblique daughter waves becomes important (see Shukla and Stenflo, 1989; Laveder et al., 2003). Linear steepening of oblique Alfvén waves can rapidly produce density perturbations which can drive the nonlinear parametric instabilities.

Another assumption in the present analysis is the use of fluid formulation. Accordingly, the important kinetic effects such as the ion Landau/cyclotron damping, (correct treatment of) heating and acceleration of the plasma and the broadening of the modulational instability regime, as mentioned earlier, are all neglected. Furthermore, inclusion of anomalously accelerated/diffused particles due to the phase coherent waves may be important (Kuramitsu and Hada, 2000; Otsuka and Hada, 2006), which are produced presumably via a parametric process, especially the modulational instabilities. In order to discuss these issues, numerical studies using two-dimensional hybrid simulations are being planned.
Acknowledgments. This paper has been supported by JSPS Research Fellowships for Young Scientists in Japan.

\section{References}

Agim, Y. Z., A. F. Vinas, and M. L. Goldstein, Magnetohydrodynamic and hybrid simulations of broadband fluctuations near interplanetary shocks, J. Geophys. Res., 100(A9), 17081-17106, 1995.

Araneda, J. A., Parametric instabilities of parallel propagating Alfvén waves: Kinetic effects in the MHD-model, Phys. Scr., T75, 164-168, 1998.

Bruno, R. and V. Carbone, The solar wind turbulece as a turbulence laboratory, Living Rev. Solar Phys., 24, 2005 [Online Article], http://www.livingreviews.org/lrsp-2005-4.

Bugnon, G., T. Passot, and P. I. Sulem, Landau-fluid simulations of Alfvén wave instabilities in a warm collisionless plasma, Nonl. Proc. Geophys., 11, 609-618, 2004.

Chanpeaux, S., D. Laveder, T. Passot, and P. L. Sulem, Remarks on the parallel propagation of small-amplitude dispersive Alfvén waves, Nonl. Proc. Geophys., 6, 169-178, 1999.

Del Zanna, L., M. Velli, and P. Londrillo, Parametric decay of circularly polarized Alfvén waves: Multidimensional simulations in periodic and open domains, $A \&$ \& , 367, 705-718, 2001.

Goldstein, M. L., J. P. Eastwood, R. A. Treumann, E. A. Lucek, J. Pickett, and P. Decreau, The near-earth solar wind, Space Sci. Rev., 118, 7-39, 2005

Hada, T., D. Koga, and E. Yamamoto, Phase coherence of MHD waves in the solar wind, Space Sci. Rev., 107(1-2), 463-466, 2003.

Hoshino, M. and M. L. Goldstein, Time evolution from linear to nonlinear stages in magnetohydrodynamic parametric instabilities, Phys. Fluids $B, 1,1405-1415,1989$.

Kuramitsu, Y. and T. Hada, Acceleration of charged particles by large amplitude MHD waves: effect of wave spatial correlation, Geophys. Res. Lett., 27(5), 629-632, 2000.

Laveder, D., T. Passot, and P. L. Sulem, Transverse dynamics of dispersive Alfven waves. I. Direct numerical evidence of filamentation, Phys. Plasmas, 9(1), 293-304, 2002.

Malara, F. and M. Velli, Parametric instability of a large-amplitude nonmonocromatic Alfvén wave, Phys. Plasmas, 3(12), 4427-4433, 1996.

Malara, F., L. Primavera, and P. Veltri, Nonlinear evolution of parametric instability of a large-amplitude nonmonocromatic Alfvén wave, Phys. Plasmas, 7(7), 2866-2877, 2000.

Nariyuki, Y. and T. Hada, Remarks on nonlinear relation among phases and frequencies in modulational instabilities of parallel propagating Alfvén waves, Nonl. Proc. Geophys., 13, 425-441, 2006 a.

Nariyuki, Y. and T. Hada, Kinetically modified parametric instabilities of circularly polarized Alfvén waves: Ion kinetic effects, Phys. Plasmas, 13, 124501, 2006b.

Narita, Y., K. H. Glassmeier and R. A. Treumann, Wave-number spectra and intermittency in the terrestrial foreshock region, Phys. Rev. Lett, 97(19), 191101, 2006

Otsuka, F. and T. Hada, Anomalous diffusion of cosmic rays in magnetic field turbulence, Prog. Theor. Phys. Suppl., 162, 204-211, 2006.

Shukla, P. K. and Stenflo, L, Filamentation instability of Alfvén waves, Astrophys. Space Sci., 155(1), 145-147, 1989.

Suzuki, T. K. and S.-I. Inutsuka, Solar winds driven by nonlinear low frequency Alfvén waves from the photosphere: Parametric study for fast/slow winds and disappearance of solar winds, J. Geophys. Res., 111, A06101, 2006.

Terasawa, T., M. Hoshino, J. I. Sakai, and T. Hada, Decay instability of finite-amplitude circulary polarized Alfvén waves: A numerical simulation of stimulated brillouin scattering, J. Geophys. Res., 91, 4171-4187, 1986.

Tsiklauri, D., J.-I., Sakai, and S. Saito, Particle-In-Cell of circularly polarized Alfvén phase mixing: A new mechanism for electron acceleration in collisionless plasmas, $A \& A, \mathbf{4 3 5}, 1105-1113,2005$.

Tu, C. Y. and E. Marsch, MHD structures, waves and turbulence in the solar-wind-observations and theories, Space Sci. Rev., 73(1-2), 1-210, 1995.

Turkmani, R. and U. Torkelsson, Propagation of non-linear circularly polarized Alfvén waves in a homogeneous medium, $A \& A, \mathbf{4 0 9}, 813-820$, 2003.

Umeki, H. and T. Terasawa, Decay instability of incoherent Alfvén waves in the solar wind, J. Geophys. Res., 97(A3), 3113-3119, 1992.

Y. Nariyuki (e-mail: nariyuki@esst.kyushu-u.ac.jp) and T. Hada 